\title{
Pharmacokinetic Modeling of Data with Below Quantification Limit
}

\author{
Madhura Dorababu* \\ Department of Pharmaceutical Sciences, College of Pharmacy, University of Tennessee Health Science Center, Memphis, TN, USA
}

Handling of data below the quantification limit (BQL) continues to be a challenge in Pharmacokinetic (PK) analyses. Such data requires sophisticated methods to evaluate the precise PK profile. PK studies are an integrated part of the development program of a new drug. They are intended to define the time course of drug and major metabolite concentrations in plasma and other biological fluids in order to obtain information on absorption, distribution, metabolism, and elimination. PK information is used to extrapolate the safety and efficacy findings to the entire patient population who may receive the New Chemical Entity (NCE) in question. Today, population PK analyses are a regular part of the documentation of an NCE. PK analysis is performed by non-compartmental (model independent) or compartmental modeling. Non-compartmental methods estimate the exposure to a drug by estimating the area under the curve of a concentration-time graph. Compartmental methods estimate the concentration-time graph using kinetic models. While population PK approach in drug development offers the possibility of gaining integrated information on pharmacokinetics, not only from relatively sparse data obtained from study subjects, but also from relatively dense data or a combination of sparse and dense data. The two common methods for population PK are i) Standard two-stage approach (STS) and ii)the nonlinear mixedeffects modeling approach (NLME). STS approach refers to fitting a pharmacokinetic model to the data of each individual; afterwards summary statistics are computed for the total collection of individual parameter estimates. Using this approach, the interindividual variance tends to be over estimated. Moreover, it is not applicable when the individual data are too sparse for individual model fits. While with NLME modeling, not only pharmacokinetic parameters but also interindividual variance parameters can be estimated. Mixed-effects or population models are more appropriate than the STS for the analysis of interindividual variation in PK.

However, the precision of the PK profile depends on the data used. PK data often contain concentration measurements below the quantification limit. While specific values cannot be assigned to these observations, nevertheless these observed BQL data are informative and generally known to be lower than the lower limit of quantification (LLQ). Setting BQLs as missing data violates the usual missing at random assumption applied to the statistical methods, and therefore leads to biased or less precise parameter estimation. By definition, these data lie within the interval $[0, \mathrm{LLQ}]$, and can be considered as censored observations. Common approaches for handling of concentration measurements reported as BQL, such as discarding the information or substitution with the quantification limit (QL) divided by two, have been shown to introduce bias to parameter estimates. Appropriate statistical methods dealing with censored data, such as maximum likelihood and Bayesian methods can be used to model PK data sets with BQL data. Beal [1] and Yang and Rogers [2] evaluated different methods for dealing with BQL data and investigate the impact of the amount of BQL observations on the bias and precision of parameter estimates in PK models under maximum likelihood method as implemented in NONMEM and a Bayesian approach using Markov chain Monte Carlo (MCMC) as applied in Win BUGS.

The methods used for the evaluation of PK data with BQL are i) discard all the BQL values $i$ ) replace all the BQL observations with QL/2 iii) replace all the BQL observations with 0 iv) each BQL observation $x$ is replaced by $\mathrm{QL} / 2$, except that any and all consecutive $\mathrm{BQL}$ observations succeeding $x$ are discarded $v$ ) discard the BQL observations, and under the assumption that all the $D(t)$ are normal, the method of maximum conditional likelihood estimation can be applied to the remaining observations vi) handle the BQL observations as fixed-point censored observations; the maximum likelihood estimation method is used to fit the PK model to all the observations, and the likelihoods forthe BQL observations in particular are taken to be the likelihoods that these observations are indeed BQL vii) similar to method vi) however it includes an adjustment to recognize that a measurement cannot really be negative viii) Bayesian approach using Markov chain Monte Carlo.

The first three methods are simpler and more familiar than the other methods. A potential problem in discarding the BQL observation is that the lower of the remaining observations misrepresent the true lower concentrations; the lower remaining observations are selectively too high. This can create a bias in the fit; hence this method clearly biases the parameter estimates, and therefore the parameter predictions. Replacement methods (ii and iii) are useful when there are very few observations, e.g., one early observation and one late observation. When the BQL values replaced by 0 (method iii), the BQL "replacement observations"are always too low, again creating a bias. If one desires to use a simple method, then when the frequency of BQL observations is small, method which discards the BQL observations (method $i$ ) is better than any other such method. Method $i v$ should only be used when it can be assumed that consecutive BQL observations reflect true concentrations that are decreasing. Using method $v$, the true lower concentrations are not misrepresented; the fact that the observed lower concentrations are obtained selectively is explicitly taken into account. With this method, the likelihood for the data, conditional onthe fact that by design, all (remaining) observations are above the QL, is maximized with respect to the model parameters. Method vi allows the BQL observations to be retained, but handles these as censored observations, under the assumption that all the $D(t)$

*Corresponding author: Dr. Madhura Dorababu, MPharm, PhD, MDBA, Department of Pharmaceutical Sciences, College of Pharmacy, University of Tennessee Health Science Center, Memphis, TN 38163, USA, Tel: + 1 6122036739; E-mail: madhuradorababu@yahoo.com

Received February 21, 2012; Accepted February 25, 2012; Published February 27, 2012

Citation: Dorababu M (2012) Pharmacokinetic Modeling of Data with Below Quantification Limit J Bioequiv Availab 4: ii-iii. doi:10.4172/jbb.10000e12

Copyright: (c) 2012 Dorababu M. This is an open-access article distributed under the terms of the Creative Commons Attribution License, which permits unrestricted use, distribution, and reproduction in any medium, provided the original author and source are credited. 
are normal. The true lower concentrations are not misrepresented with this method. Simple substitution methods were problematic where a high proportion of BQLs were in the data. Methods using maximum likelihood estimations are more rational than the simple methods like replacement of BQL values with QL/2. A likelihood-based method as implemented in NONMEM was shown to have advantages over other simple substitution methods. Use of MCMC based Bayesian approach also has advantageous. One of which is that it is easy to calculate the posterior distribution for any other derived parameter. For example, in the situation of linear $\mathrm{PK}$, total area under the concentration-time curve $(\mathrm{AUC}=\mathrm{D} / \mathrm{CL})$ is usually an important parameter in correlating exposure with safety signals or clinical benefits. The posterior distribution of AUC as a parameter is formed by simply calculating the derived parameter $(\mathrm{AUC}=\mathrm{D} / \mathrm{CL})$ for each sample from the posterior of CL. Analyses that maximized the likelihood of the data above the limit of quantification and treated BQL data as censored provided the most accurate and precise parameter estimates.

\section{References}

1. Beal SL (2001) Ways to fit a PK model with some data below the quantification limit. J Pharmacokinet Pharmacodyn 28: 481-504.

2. Yan S, RogerJ (2010) Evaluations of Bayesian and maximum likelihood methods in PK models with below-quantification-limit data. Pharm Stat 9 : 313-330. 